\title{
Boolean Game with Prioritized Norms
}

\author{
Xin Sun \\ Faculty of Science, Technology and Communication, University of Luxembourg \\ xin.sun@uni.lu
}

\begin{abstract}
In this paper we study boolean game with prioritized norms. Norms distinguish illegal strategies from legal strategies. Notions like legal strategy and legal Nash equilibrium are introduced. Our formal model is a combination of (weighted) boolean game and so called (prioritized) input/output logic. After formally presenting the model, we use examples to show that non-optimal Nash equilibrium can be avoided by making use of norms. We study various complexity issues related to legal strategy and legal Nash equilibrium.
\end{abstract}

Key words: boolean game, norm, input/output logic

\section{Introduction}

The study of the interplay of games and norms can be divided into two main branches: the first, mostly originating from economics and game theory [11, $19,20]$, treats norms as mechanisms that enforce desirable properties of social interactions; the second, that has its roots in social sciences and evolutionary game theory $[29,12]$ views norms as (Nash or correlated) equilibrium that results from the interaction of rational agents. A survey of the interaction between games and norms can be found in Grossi et al [15]. This paper belongs to the first branch.

In this paper we study the combination of boolean games and norms. Boolean game is a class of games based on propositional logic. It was firstly introduced by Harrenstein et al. [17] and further developed by several researchers [16, 23, 13, $9,7,26]$. In a boolean game, each agent $i$ is assumed to have a goal, represented by a propositional formula $\phi_{i}$ over some set of propositional variables $\mathbb{P}$. Each agent $i$ is associated with some subset $\mathbb{P}_{i}$ of the variables, which are under the unique control of agent $i$. The choices, or strategies, available to $i$ correspond to all the possible assignment of truth or falsity to the variables in $\mathbb{P}_{i}$. An agent will try to choose an assignment so as to satisfy his goal $\phi_{i}$. Strategic concerns arise because whether $i$ 's goal is in fact satisfied will depend on the choices made by other agents.

Norms are social rules regulating agents' behavior by prescribing which actions are obligatory, forbidden or permitted. In the game theoretical setting, norms distinguish illegal strategies form legal strategies. By designing norms appropriately, non-optimal equilibrium might be avoided. To represent norms in 
boolean games, we need a logic of norms, which has been extensively studied in the deontic logic community.

Various deontic logic has been developed since von Wright's first paper [30] in this area. In the first volume of the handbook of deontic logic [14], input/output logic $[21,22]$ appears as one of the new achievement in deontic logic in recent years. Input/output logic takes its origin in the study of conditional norms. The basic idea is: norms are conceived as a deductive machine, like a black box which produces normative statements as output, when we feed it factual statements as input.

In this paper we use a simplification of Parent's prioritized input/output logic [25] as the logic of norms. Given a normative multi-agent system, which contains a boolean game, a set of prioritized norms and certain environment. Every strategy of every agent is classified as legal or illegal. Notions like legal Nash equilibrium are then naturally defined.

The structure of this paper is the following: We present some background knowledge, including boolean game, input/output logic and complexity theory in Section 2. Normative multi-agent system are introduced and its complexity issues are studied in Section 3. We conclude this paper in Section 4.

\section{Background}

\subsection{Propositional logic}

Let $\mathbb{P}=\left\{p_{0}, p_{1}, \ldots\right\}$ be a finite set of propositional variables and let $L_{\mathbb{P}}$ be the propositional language built from $\mathbb{P}$ and boolean constants $T$ (true) and $\perp$ (false) with the usual connectives $\neg, \vee, \wedge, \rightarrow$ and $\leftrightarrow$. Formulas of $L_{\mathbb{P}}$ are denoted by $\phi, \psi$ etc. A literal is a variable $p \in \mathbb{P}$ or its negation. $2^{\mathbb{P}}$ is the set of the valuations for $\mathbb{P}$, with the usual convention that for $V \in 2^{\mathbb{P}}$ and $p \in V, V$ gives the value true to $p$ if $p \in V$ and false otherwise. $\vDash$ denotes the classical logical consequence relation.

Let $X \subseteq \mathbb{P}, 2^{X}$ is the set of $X$-valuations. A partial valuation (for $\mathbb{P}$ ) is an $X$-valuation for some $X \subseteq \mathbb{P}$. Partial valuations are denoted by listing all variables of $X$, with a " +" symbol when the variable is set to be true and a " - " symbol when the variable is set to be false: for instance, let $X=\{p, q, r\}$, then the $X$-valuation $V=\{p, r\}$ is denoted $\{+p,-q,+r\}$. If $\left\{\mathbb{P}_{1}, \ldots, \mathbb{P}_{n}\right\}$ is a partition of $\mathbb{P}$ and $V_{1}, \ldots, V_{n}$ are partial valuations, where $V_{i} \in 2^{\mathbb{P}_{i}},\left(V_{1}, \ldots, V_{n}\right)$ denotes the valuation $V_{1} \cup \ldots \cup V_{n}$.

\subsection{Boolean game}

Boolean games introduced by Harrenstein et al [17] are zero-sum games with two players, where the strategies available to each player consist in assigning a truth value to each variable in a given subset of $\mathbb{P}$. Bonzon et al [8] give a more general definition of a boolean game with any number of players and not necessarily zero-sum. In this paper we further generalizes boolean games such 
that the utility of each agent is not necessarily in $\{0,1\}$. Such generalization is reached by representing the goals of each agent as a set of weighted formulas. We call such boolean game weighted boolean game. The idea of using weighted formulas to define utility can be found in many work among which we mention satisfiability game [5] and weighted boolean formula game [23].

Definition 1 (boolean game). A weighted boolean game is a 4-tuple (Agent, $\mathbb{P}, \pi$, Goal), where

1. Agent $=\{1, \ldots, n\}$ is a set of agents.

2. $\mathbb{P}$ is a finite set of propositional variables.

3. $\pi:$ Agent $\mapsto 2^{\mathbb{P}}$ is a control assignment function such that $\{\pi(1), \ldots, \pi(n)\}$ forms a partition of $\mathbb{P}$. For each agent $i, 2^{\pi(i)}$ is the strategy space of $i$.

4. Goal $=\left\{\right.$ Goal $_{1}, \ldots$, Goal $\left._{n}\right\}$ is a set of weighted formulas of $L_{\mathbb{P}}$. That is, each Goal $_{i}$ is a finite set $\left\{\left\langle\phi_{1}, m_{1}\right\rangle, \ldots,\left\langle\phi_{k}, m_{k}\right\rangle\right\}$ where $\phi_{j} \in L_{\mathbb{P}}$ and $m_{j}$ is a real number.

A strategy for agent $i$ is a partial valuation for all the variables $i$ controls. Note that since $\{\pi(1), \ldots, \pi(n)\}$ forms a partition of $\mathbb{P}$, a strategy profile $S$ is a valuation for $\mathbb{P}$. In the rest of the paper we make use of the following notation, which is standard in game theory. Let $G=($ Agent, $\mathbb{P}, \pi$, Goal $)$ be a weighted boolean game with Agent $=\{1, \ldots, n\}, S=\left(s_{1}, \ldots, s_{n}\right)$ be a strategy profile. $s_{-i}$ denotes the projection of $S$ on Agent $-\{i\}: s_{-i}=\left(s_{1}, \ldots, s_{i-1}, s_{i+1}, \ldots, s_{n}\right)$.

Agents' utilities in weighted boolean games are induced by their goals. For every agent $i$ and every strategy profiles $S, u_{i}(S)=\Sigma\left\{m_{j}:\left\langle\phi_{j}, m_{j}\right\rangle \in\right.$ Goal $_{i}, S \vDash$ $\left.\phi_{j}\right\}$. Dominating strategies and pure-strategy Nash equilibria are defined as usual in game theory [24].

Example 1 Let $G=($ Agent, $\mathbb{P}, \pi$, Goal $)$ where Agent $=\{1,2\}, \mathbb{P}=\{p, q, s\}$, $\pi(1)=\{p\}, \pi(2)=\{q, s\}$, Goal $_{1}=\{\langle p \leftrightarrow q, 1\rangle,\langle s, 2\rangle\}$, Goal $_{2}=\{\langle p \wedge q, 2\rangle,\langle\neg s, 1\rangle\}$. This boolean game is depicted as follows:

\begin{tabular}{|c|c|c|c|c|}
\hline & $+q,+s$ & $+q,-s$ & $-q,+s$ & $-q,-s$ \\
\hline$+p$ & $(3,2)$ & $(1,3)$ & $(2,0)$ & $(0,1)$ \\
\hline$-p$ & $(2,0)$ & $(0,1)$ & $(3,0)$ & $(1,1)$ \\
\hline
\end{tabular}

\subsection{Input/output logic}

In input/output logic, a norm is an ordered pair of formulas $(\phi, \psi) \in L_{\mathbb{P}} \times L_{\mathbb{P}}$, which is read as "given $\phi$, it is obligatory to be $\psi$ ". A set of norm $N$ can be viewed as a function from $2^{L_{\mathbb{P}}}$ to $2^{L_{\mathbb{P}}}$ such that for a set $\Phi$ of formulas, $N(\Phi)=\left\{\psi \in L_{\mathbb{P}}:(\phi, \psi) \in N\right.$ for some $\left.\phi \in \Phi\right\}$. A finite set of norms is called a (plain) normative system. 
Definition 2 (Semantics of input/output logic [21]). Given a normative system $N$ and a finite set of formulas $\Phi$, out $(N, \Phi)=C n(N(C n(\Phi)))$, where $C n$ is the consequence relation of propositional logic. ${ }^{1}$

Intuitively, the procedure of the semantics is as follows: We first have in hand a set of formulas $\Phi$ (call it the input) as a description of the current state. We then close it by logical consequence $C n(\Phi)$. The set of norms, like a deductive machine, accepts this logically closed set and produces a set of formulas $N(C n(\Phi))$. We finally get the output $C n(N(C n(\Phi)))$ by applying the logical closure again. $\psi \in$ $\operatorname{out}(N, \Phi)$ is understood as " $\psi$ is obligatory given facts $\Phi$ and norms $N$ ".

Example 2 Let $p, q, r$ are propositional variables. Let $N=\{(p, q),(p \vee q, r),(r, p)\}$. Then $\operatorname{out}(N,\{p\})=C n(N(C n(\{p\})))=C n(\{q, r\})$.

Input/output logic is given a proof theoretic characterization. We say that an ordered pair of formulas is derivable from a set $N$ iff $(a, x)$ is in the least set that extends $N$ and is closed under a number of derivation rules. The following are the rules we need:

- SI (strengthening the input): from $(\phi, \psi)$ to $(\chi, \psi)$ whenever $\chi \vDash \phi$.

- WO (weakening the output): from $(\phi, \psi)$ to $(\phi, \chi)$ whenever $\psi \vDash \chi$.

- AND (conjunction of output): from $(\phi, \psi)$ and $(\phi, \chi)$ to $(\phi, \psi \wedge \chi)$.

The derivation system based on the rules SI, WO and AND is denoted as $\operatorname{deriv}(N)$.

Example 3 Let $N=\{(p \vee q, r),(q, r \rightarrow s)\}$, then $(q, s) \in \operatorname{deriv}(N)$ because we have the following derivation

1. $(p \vee q, r)$

Assumption

2. $(q, r)$

1, SI

3. $(q, r \rightarrow s)$

Assumption

4. $(q, r \wedge(r \rightarrow s))$

2,3, $A N D$

5. $(q, s)$

4, WO

In Makinson and van der Torre [21], the following soundness and completeness theorem is proved:

Theorem 1 ([21]). Given a set of norms $N$,

$$
\psi \in \operatorname{out}(N,\{\phi\}) \text { iff }(\phi, \psi) \in \operatorname{deriv}(N) .
$$

\footnotetext{
${ }^{1}$ In Makinson and van der Torre [21], this logic is called simple-minded input/output logic. Different input/output logics are developed in Makinson and van der Torre [21] as well. A technical introduction of input/output logic can be found in Sun [28].
} 
Prioritized input/output logic A prioritized normative system $N \geq=(N, \geq)$ is a finite set of norms together with a priority relation over norms. We assume $\geq$ to be reflexive and transitive and understand $(\phi, \psi) \geq\left(\phi^{\prime}, \psi^{\prime}\right)$ as $(\phi, \psi)$ has higher priority than $\left(\phi^{\prime}, \psi^{\prime}\right)$. The priority relation is further lifted to priority over sets of norms. Following Parent [25], we define the lifting as follows: $N_{1} \succeq N_{2}$ iff for all $\left(\phi_{2}, \psi_{2}\right) \in N_{2}-N_{1}$ there is $\left(\phi_{1}, \psi_{1}\right) \in N_{1}-N_{2}$ such that $\left(\phi_{1}, \psi_{1}\right) \geq\left(\phi_{2}, \psi_{2}\right)$.

Definition 3 (output with priorities ${ }^{2}$ ). Let $N^{\geq}$be a prioritized normative system and $\Phi$ be a set of formulas.

$$
\psi \in \operatorname{out}_{p}\left(N^{\geq}, \Phi\right) \text { iff } \psi \in \bigcap\left\{\operatorname{out}\left(N^{\prime}, \Phi\right): N^{\prime} \in \operatorname{preffamily}\left(N^{\geq}, \Phi\right)\right\} .
$$

Here preffamily $\left(N^{\geq}, \Phi\right)$ is defined via the following steps:

1. maxfamily $\left(N^{\geq}, \Phi\right)$ is the set of $\subseteq$-maximal subsets $N^{\prime}$ of $N$ such that out $\left(N^{\prime}, \Phi\right)$ is consistent. That is, out $\left(N^{\prime}, \Phi\right)$ is consistent and for all $N^{\prime \prime}$ such that $N^{\prime} \subset N^{\prime \prime}$, out $\left(N^{\prime \prime}, \Phi\right)$ is not consistent

2. filterfamily $\left(N^{\geq}, \Phi\right)$ is the set of norms $N^{\prime} \in \max f a m i l y\left(N^{\geq}, \Phi\right)$ that maximize the output, i.e., that are such that out $\left(N^{\prime}, \Phi\right) \subset$ out $\left(N^{\prime \prime}, \Phi\right)$ for no $N^{\prime \prime} \in \max \operatorname{amily}\left(N^{\geq}, \Phi\right)$.

3. preffamily $\left(N^{\geq}, \Phi\right)$ is the set of $\succeq$-maximal elements of filterfamily $\left(N^{\geq}, \Phi\right)$.

Permission in input/output logic Philosophically, it is common to distinguish between two kinds of permission: negative permission and positive permission. Negative permission is straightforward to describe: something is negatively permitted according to certain norms iff it is not prohibited by those norms. That is, iff there is no obligation to the contrary. Positive permission is more elusive. For the sake of simplicity, in this paper when only discuss negative permission and leave other types of permission as future work.

Definition 4 (permission). Given a prioritized normative system $N \geq$ and a finite set of formulas $\Phi, \operatorname{Perm}\left(N^{\geq}, \Phi\right)=\left\{\psi \in L_{\mathbb{P}}: \neg \psi \notin\right.$ out $\left._{p}\left(N^{\geq}, \Phi\right)\right\}$.

Intuitively, $\phi$ is permitted iff $\phi$ is not forbidden. Since a formula is forbidden iff its negation is obligatory, $\phi$ is not forbidden is equivalent to $\neg \phi$ is not obligatory.

\subsection{Complexity theory}

Complexity theory is the theory to investigate the time, memory, or other resources required for solving computational problems. In this subsection we briefly review those concepts and results from complexity theory which will be used in this paper. More comprehensive introduction of complexity theory can be found in [4]

We assume the readers are familiar with notions like Turing machine and the complexity class P, NP and coNP. Oracle Turing machine and two complexity classes related to oracle Turing machine will be used in this paper.

\footnotetext{
${ }^{2}$ Here our prioritized input/output logic is a simplification of the original version of Parent [25].
} 
Definition 5 (oracle Turing machine [4]). An oracle for a language $L$ is a device that is capable of reporting whether any string $w$ is a member of L. An oracle Truing machine $M^{L}$ is a modified Turing machine that has the additional capability of querying an oracle. Whenever $M^{L}$ writes a string on a special oracle tape it is informed whether that string is a member of $L$, in a single computation step.

$\mathrm{P}^{N P}$ is the class of problems solvable by a deterministic polynomial time Turing machine with an NP oracle. $\mathrm{P}^{N P[O(\log n)]}$ only allows $O(\log n)$ oracle queries instead of polynomially-many. $\mathrm{P}_{\|}^{N P}$ is the class of problems which can be solved by using the $N P$ oracle only in parallel. Buss and Hay [10] show that $\mathrm{P}_{\|}^{N P}$ coincide with $\mathrm{P}_{\| O(1)}^{N P}$, where a fixed number of parallel rounds is allowed.

$\mathrm{NP}^{N P}$ is the class of problems solvable by a non-deterministic polynomial time Turing machine with an NP oracle. Another name for the class $\mathrm{NP}^{N P}$ is $\Sigma_{2}^{p} . \Sigma_{i+1}^{p}$ is the class of problems solvable by a non-deterministic polynomial time Turing machine with a $\Sigma_{i}^{p}$ oracle. $\Pi_{i}^{p}$ is the class of problems of which the complement is in $\Sigma_{i}^{p}$.

\section{From boolean game to normative multi-agent system}

In recent years, normative multi-agent system $[6,3]$ arises as a new interdisciplinary academic area bringing together researchers from multi-agent system $[27$, $32,31]$, deontic logic [14] and normative system $[1,18,2]$. By combining boolean games and norms, we here develop a new approach to normative multi-agent system.

Definition 6 (normative multi-agent system). A normative multi-agent system is a triple $\left(G, N^{\geq}, E\right)$ where

- $G=($ Agent $, \mathbb{P}, \pi$, Goal $)$ is a weighted boolean game.

- $N \geq$ is a prioritized normative system.

$-E \subseteq L_{\mathbb{P}}$ is a finite set of formulas representing the environment.

\subsection{Legal strategy}

In a normative multi-agent system, agent's strategies are classified as either legal or illegal. The basic idea is viewing strategies as formulas and using the mechanism of input/output logic to decide whether a formula is permitted.

Definition 7 (legal strategy). Given a normative multi-agent system $(G, N \geq, E)$, for each agent $i$, a strategy $\left(+p_{1}, \ldots,+p_{m},-q_{1}, \ldots,-q_{n}\right)$ is legal if

$$
p_{1} \wedge \ldots \wedge p_{m} \wedge \neg q_{1} \wedge \ldots \wedge \neg q_{n} \in \operatorname{Perm}\left(N^{\geq}, E\right) .
$$

Example 4 Consider the prisoner's dilemma augmented with norms. Let $\left(G, N^{\geq}, E\right)$ be a normative multi-agent system as following: 
- $G=($ Agent $, \mathbb{P}, \pi$, Goal $)$ is a weighted boolean game with

- Agent $=\{1,2\}$,

- $\mathbb{P}=\{p, q\}$,

- $\pi(1)=\{p\}, \pi(2)=\{q\}$,

- Goal $_{1}=\{\langle p, 2\rangle,\langle\neg q, 3\rangle\}$, Goal $_{2}=\{\langle q, 2\rangle,\langle\neg p, 3\rangle\}$.

- $N^{\geq}=(N, \geq)$ where $N=\{(\top, \neg p),(\top, \neg q),(\top, q)\},(\top, \neg q) \geq(\top, q)$.

$-E=\emptyset$.

\begin{tabular}{|c|c|c|}
\hline & $+q$ & $-p$ \\
\hline$+p$ & $(2,2)$ & $(5,0)$ \\
\hline$-p$ & $(0,5)$ & $(3,3)$ \\
\hline
\end{tabular}

Then out $(N, E)=C n(\{\neg p, \neg q, q\}), \max \operatorname{family}\left(N^{\geq}, E\right)=\{\{(\top, \neg p),(\top, \neg q)\}$, $\{(\top, \neg p),(\top, q)\}\}$, filterfamily $\left(N^{\geq}, E\right)=\max f a m i l y\left(N^{\geq}, E\right)$, preffamily $\left(N^{\geq}\right.$, $E)=\{\{(\top, \neg p),(\top, \neg q)\}\}$. Therefore out $\left(N^{\geq}, E\right)=\operatorname{out}(\{(\top, \neg p),(\top, \neg q)\}, E)=$ $C n(\{\neg p, \neg q\})$.

Therefore $\{-p\}$ and $\{-q\}$ are legal while $\{+p\}$ and $\{+q\}$ are not.

Having defined the notion legal strategy, a natural question to ask is how complex is it to decide whether a strategy is legal. Theorem 2 gives a first answer to this question. To prove Theorem 2, we need the following lemmas.

Lemma 1. Given a normative system $N$, a finite set of formulas $\Phi$ and a formula $\phi$, deciding whether $\phi \in \operatorname{out}(N, \Phi)$ is coNP hard and in $P_{\|}^{N P}$.

Proof. Concerning the coNP hardness, we prove by reducing the validity problem of propositional logic to our problem. Let $\phi$ be an arbitrary formula. Let $N=\emptyset$ and $\Phi=\emptyset$, then $\phi$ is a tautology iff $\phi \in C n(\top)$ iff $\phi \in C n(N(C n(\Phi)))$ iff $\phi \in \operatorname{out}(N, \Phi)$

Concerning the $P_{\|}^{N P}$ membership, we prove by giving an oracle Turing machine with oracle $S A T$, the set of all satisfiable propositional formulas, to solve this problem.

Let $N=\left\{\left(\phi_{1}, \psi_{1}\right), \ldots,\left(\phi_{n}, \psi_{n}\right)\right\}$.

1. for each $\phi_{i} \in\left\{\phi_{1}, \ldots, \phi_{n}\right\}$, use the oracle to test if $\Phi \vDash \phi_{i}$.

(a) If yes, then mark $\psi_{i}$,

(b) Otherwise do nothing.

2. Let $\psi_{i_{1}}, \ldots \psi_{i_{k}}$ be all those $\psi_{i}$ which are marked in step 1 .

3. Use the oracle to test if $\left\{\psi_{i_{1}}, \ldots, \psi_{i_{k}}\right\} \vDash \phi$.

(a) If yes, then return "accept"

(b) Otherwise return "reject". 
It can be verified that $\phi \in C n(N(C n(\Phi)))$ iff the Turing machine returns "accept" and the time complexity of the oracle Turing machine runs in polynomial time and calls the oracle in parallel for 2 rounds. Therefore the problem is in $P_{\| O(1)}^{N P}$, which coincides with $P_{\|}^{N P}$.

Lemma 2. Given a prioritized normative system $N \geq$, a finite set of norms $N^{\prime} \subseteq N$, a finite set of formulas $\Phi$, deciding whether $N^{\prime} \in \max f \operatorname{mily}(N \geq, \Phi)$ is coNP hard and in $P^{N P}$.

Proof. The coNP hardness is easy to prove. Here we focuses on the $P^{N P}$ membership. We prove by giving an oracle Turing machine with oracle $S A T$ to solve this problem.

Let $N-N^{\prime}=\left\{\left(\phi_{1}, \psi_{1}\right), \ldots,\left(\phi_{n}, \psi_{n}\right)\right\}$.

1. Test if $\perp \in \operatorname{out}\left(N^{\prime}, \Phi\right)$.

2. If yes, return "reject". Otherwise continue.

3. For all $i \in\{1, \ldots, n\}$, test if $\perp \in \operatorname{out}\left(N^{\prime} \cup\left\{\left(\phi_{i}, \psi_{i}\right)\right\}, \Phi\right)$.

4. Return "accept" if $\perp \in$ out $\left(N^{\prime} \cup\left\{\left(\phi_{i}, \psi_{i}\right)\right\}, \Phi\right)$ for all $i \in\{1, \ldots, n\}$. Otherwise return "reject".

It can be verified that $N^{\prime} \in \max \operatorname{family}\left(N^{\geq}, \Phi\right)$ iff the Turing machine returns "accept" and the time complexity of the oracle Turing machine is polynomial. $\dashv$

Lemma 3. Given a prioritized normative system $N \geq$, a finite set of norms $N^{\prime} \subseteq N$, a finite set of formulas $\Phi$, deciding whether $N^{\prime} \in \operatorname{filterfamily}\left(N^{\geq}, \Phi\right)$ is coNP hard and in $\operatorname{coN} P^{N P}=\Pi_{2}^{p}$.

Proof. The coNP hardness is easy to prove. Here we focuses on the $c o N P^{N P}$ membership.

We prove by giving a non-deterministic oracle Turing machine with oracle $S A T$ to solve the complement of this problem.

1. Test if $N^{\prime} \in \max f a m i l y\left(N^{\geq}, \Phi\right)$. If no, return "accept". Otherwise continue.

2. Guess a set of norms $N^{\prime \prime} \subseteq N$.

3. Test if $N^{\prime \prime} \in \operatorname{maxfamily}\left(N^{\geq}, \Phi\right)$. If no, return "reject" on this branch. Otherwise continue.

4. Test if $\vDash \wedge N^{\prime \prime}(C n(\Phi)) \rightarrow \bigwedge N^{\prime}(C n(\Phi))$ meanwhile $\not \models \wedge N^{\prime}(C n(\Phi)) \rightarrow$ $\bigwedge N^{\prime \prime}(C n(\Phi))$. If yes, return "accept" on this branch. Otherwise return "reject" on this branch.

It can be verified that $N^{\prime} \notin$ filterfamily $\left(N^{\geq}, \Phi\right)$ iff the non-deterministic Turing machine returns "accept" on some branches. The time complexity of the non-deterministic Turing machine is polynomial because with the help of an NP oracle $S A T$, the test in step 1,3 and 4 can be done in polynomial time.

Lemma 4. Given a prioritized normative system $N \geq$, a finite set of norms $N^{\prime} \subseteq N$, a finite set of formulas $\Phi$, deciding whether $N^{\prime} \in \operatorname{preffamily}\left(N^{\geq}, \Phi\right)$ is coNP hard and in $\Pi_{3}^{p}$. 
Proof. The hardness is easy to prove. Here we focus on the membership. We prove by giving a non-deterministic oracle Turing machine with a $\Sigma_{2}^{p}$ oracle to solve the complement of this problem.

1. Test if $N^{\prime} \in \operatorname{filterfamily}\left(N^{\geq}, \Phi\right)$. If no, return "accept" on this branch. Otherwise continue.

2. Guess a set of norms $N^{\prime \prime} \subseteq N$.

3. Test if $N^{\prime \prime} \in$ filterfamily $\left(N^{\geq}, \Phi\right)$. If no, return "reject" on this branch. Otherwise continue.

4. Test if $N^{\prime \prime} \succeq N^{\prime}$. If yes, return "accept" on this branch. Otherwise return "reject" on this branch.

It can be verified that $N^{\prime} \notin \operatorname{preffamily}\left(N^{\geq}, \Phi\right)$ iff the non-deterministic Turing machine returns "accept" on some branch. The time complexity of the non-deterministic Turing machine is polynomial because with the help of an $\Sigma_{2}^{p}$ oracle, the test in step 1 and 3 can be done in polynomial time.

Theorem 2. Given a normative multi-agent system $\left(G, N^{\geq}, E\right)$ and a strategy $\left(+p_{1}, \ldots,+p_{m},-q_{1}, \ldots,-q_{n}\right)$, deciding whether this strategy is legal is $N P$ hard and in $\Pi_{4}^{p}$.

Proof. To show that this problem is NP hard, we provide a reduction from the satisfiability problem of propositional logic to the problem of deciding whether a strategy is legal.

Let $\phi$ be a formula. Let $s=\{+p\}$ be a strategy, $N=\{(\neg \phi, \neg p)\}, E=\emptyset$. We will show $\phi$ is satisfiable iff $s$ is legal.

Recall that $p \in \operatorname{Perm}(N, E)$ iff $\neg p \notin \operatorname{out}_{p}(N, E)$. In this case we have $\operatorname{out}_{p}(N, E)=\operatorname{out}(N, E)=C n(N(C n(E)))$.

From $E=\emptyset$ we know that $C n(N(C n(E)))=C n(N(C n(\top)))$. Therefore if $\phi$ is satisfiable, then $\neg \phi$ is not a tautology. Therefore $N(C n(E))=\emptyset$. Hence $\neg p \notin C n(N(C n(E)))=\operatorname{out}(O, E), p \in \operatorname{Perm}(N, E)$. If $\phi$ is not satisfiable, then $\neg \phi$ is a tautology. Hence $\neg p \in N(C n(E)) \subseteq C n(N(C n(E)))=\operatorname{out}(N, E)$, which means $p \notin \operatorname{Perm}(N, E)$.

For the $\Pi_{4}^{p}$ membership, we prove by giving a non-deterministic oracle Turing machine with an $\Sigma_{3}^{p}$ oracle to solve the complement of this problem.

1. Guess a set of norms $N^{\prime} \subseteq N$.

2. Test if $N^{\prime} \in$ preffamily $\left(N^{\geq}, \Phi\right)$. If no, return "reject" on this branch. Otherwise continue.

3. Test if $\neg\left(p_{1} \wedge \ldots \wedge p_{m} \wedge \neg q_{1} \wedge \ldots \wedge \neg q_{n}\right) \in \operatorname{out}\left(N^{\prime}, E\right)$. If yes, return "accept" on this branch.

It can be verified that $p_{1} \wedge \ldots \wedge p_{m} \wedge \neg q_{1} \wedge \ldots \wedge \neg q_{n} \notin$ out $p_{p}(N, E)$ iff the non-deterministic Turing machine returns "accept" on some branch and the time complexity of the Turing machine is polynomial. 


\subsection{Legal Nash equilibrium}

A (pure-strategy) legal Nash equilibrium is a strategy profile which contains only legal strategies and no agent can improve his utility by choosing another legal strategy, given others do not change their strategies.

Definition 8 (Legal Nash equilibrium). Given a normative multi-agent system $(G, N, E)$, A strategy profile $S=\left(s_{1}, \ldots, s_{n}\right)$ is a legal Nash equilibrium if

- for every agent $i, s_{i}$ is a legal strategy

- for every agent $i$, for every legal strategy $s_{i}^{\prime} \in S_{i}, u_{i}(S) \geq u_{i}\left(s_{i}^{\prime}, s_{-i}\right)$.

Example 5 In the normative multi-agent system presented in Example $4,(-p,-q)$ is the unique legal Nash equilibria.

Example 6 Let $(G, N \geq, E)$ be a normative system as following:

- $G=($ Agent $, \mathbb{P}, \pi, G o a l)$ is a weighted boolean game with

- Agent $=\{1,2\}$,

- $\mathbb{P}=\{p, q\}$,

- $\pi(1)=\{p\}, \pi(2)=\{q\}$,

- Goal $_{1}=$ Goal $_{2}=\{\langle p \wedge q, 2\rangle,\langle\neg p \wedge \neg q, 3\rangle\}$.

$-N^{\geq}=(N, \geq), N=\{(\top, \neg p),(\top, \neg q)\},(\top, \neg p) \geq(\top, \neg q),(\top, \neg q) \geq(\top, \neg p)$.

$-E=\emptyset$.

\begin{tabular}{|c|c|c|}
\hline & $+q$ & $-p$ \\
\hline$+p$ & $(2,2)$ & $(0,0)$ \\
\hline$-p$ & $(0,0)$ & $(3,3)$ \\
\hline
\end{tabular}

Without normative system there are two Nash equilibrium: $(+p,+q)$ and $(-p,-q)$. There is only one legal Nash equilibria: $(-p,-q)$. From the perspective of social welfare, $(+p,+q)$ is not an optimal equilibria because its social welfare is $2+2=4$, while the social welfare of $(-p,-q)$ is $3+3=6$. Therefore this example shows that by designing norms appropriately, non-optimal equilibrium might be avoided

Theorem 3. Given a normative multi-agent system $(G, N \geq, E)$ and a strategy profile $S=\left(s_{1}, \ldots, s_{n}\right)$. Deciding whether $S$ is a legal Nash equilibrium is $N P$ hard and in $\Pi_{5}^{p}$.

Proof. The NP hardness is trivial. For the $\Pi_{5}^{p}$ membership, we prove by giving a non-deterministic oracle Turing machine with a $\Sigma_{4}^{p}$ oracle to solve the complement of this problem.

1. Test if $S$ is legal. If no, return "accept". Otherwise continue. 
2. Guess a strategy profile $S^{\prime}$

3. Test if $S^{\prime}$ is legal. If no, return "reject" on this branch. Otherwise continue.

4. For each agent $i$, test if $u_{i}(S)<u_{i}\left(S^{\prime}\right)$. Return "accept" on this branch if for some $i, u_{i}(S)<u_{i}\left(S^{\prime}\right)$. Otherwise return "reject" on this branch.

It can be verified that $S$ is not a legal Nash equilibrium iff the non-deterministic Turing machine returns "accept" on some branch and the time complexity of the Turing machine is polynomial.

Theorem 4. Given a normative multi-agent system $(G, N, E)$. Deciding whether there is a legal Nash equilibrium of $G$ is $\Sigma_{2}^{P}$ hard and in $\Sigma_{6}^{P}$.

Proof. The lower bound follows from the fact that deciding whether there is a Nash equilibria for boolean games without norms is $\Sigma_{2}^{P}$ complete [8]. Concerning the upper bound, recall that $\Sigma_{6}^{P}=N P^{\Sigma_{5}^{P}}$. The problem can be solved by a polynomial time non-deterministic Turing machine with an $\Sigma_{5}^{P}$ oracle.

\section{Conclusion}

In the present paper we introduce weighted boolean game with prioritized norms. Norms distinguish illegal strategies from legal strategies. Using ideas from (prioritized) input/output logic, legal strategies and legal Nash equilibrium are discussed. After formally presenting the model, we use examples to show that nonoptimal Nash equilibrium can be avoided by making use of norms. We study the complexity issues related to legal strategy and legal Nash equilibrium. Our complexity results are not complete, which leaves rooms for future work. Other natural future work includes using a different input/output logic to reason about norms and using positive permission to define legal strategy.

\section{References}

1. Thomas Ågotnes, Wiebe van der Hoek, Juan A. Rodríguez-Aguilar, Carles Sierra, and Michael Wooldridge. On the logic of normative systems. In Manuela M. Veloso, editor, IJCAI 200\%, Proceedings of the 20th International Joint Conference on Artificial Intelligence, Hyderabad, India, January 6-12, 2007, pages 1175-1180, 2007.

2. Natasha Alechina, Mehdi Dastani, and Brian Logan. Reasoning about normative update. In Francesca Rossi, editor, IJCAI 2013, Proceedings of the 23rd International Joint Conference on Artificial Intelligence, Beijing, China, August 3-9, 2013. IJCAI/AAAI, 2013.

3. Giulia Andrighetto, Guido Governatori, Pablo Noriega, and Leendert W. N. van der Torre, editors. Normative Multi-Agent Systems, volume 4 of Dagstuhl Follow-Ups. Schloss Dagstuhl - Leibniz-Zentrum fuer Informatik, 2013.

4. Sanjeev Arora and Boaz Barak. Computational Complexity: A Modern Approach. Cambridge University Press, New York, USA, 2009. 
5. Vittorio Bilò. On satisfiability games and the power of congestion games. In MingYang Kao and Xiang-Yang Li, editors, Algorithmic Aspects in Information and Management, Third International Conference, AAIM 200\%, Portland, OR, USA, June 6-8, 2007, Proceedings, volume 4508 of Lecture Notes in Computer Science, pages 231-240. Springer, 2007.

6. Guido Boella, Leendert van der Torre, and Harko Verhagen. Introduction to the special issue on normative multiagent systems. Autonomous Agents and MultiAgent Systems, 17(1):1-10, 2008.

7. Elise Bonzon, Marie-Christine Lagasquie-Schiex, and Jérôme Lang. Dependencies between players in boolean games. Int. J. Approx. Reasoning, 50(6):899-914, 2009.

8. Elise Bonzon, Marie-Christine Lagasquie-Schiex, Jérôme Lang, and Bruno Zanuttini. Boolean games revisited. In Gerhard Brewka, Silvia Coradeschi, Anna Perini, and Paolo Traverso, editors, ECAI 2006, 17th European Conference on Artificial Intelligence, August 29 - September 1, 2006, Riva del Garda, Italy, Including Prestigious Applications of Intelligent Systems (PAIS 2006), Proceedings, volume 141 of Frontiers in Artificial Intelligence and Applications, pages 265-269. IOS Press, 2006.

9. Elise Bonzon, Marie-Christine Lagasquie-Schiex, Jérôme Lang, and Bruno Zanuttini. Compact preference representation and boolean games. Autonomous Agents and Multi-Agent Systems, 18(1):1-35, 2009.

10. Samuel R. Buss and Louise Hay. On truth-table reducibility to SAT. Inf. Comput., 91(1):86-102, 1991.

11. Ronald Coase. The problem of social cost. Journal of Law and Economics,, 1, 1960.

12. James Coleman. Foundations of Social Theory. Belnap Press, 1998.

13. Paul E. Dunne, Wiebe van der Hoek, Sarit Kraus, and Michael Wooldridge. Cooperative boolean games. In Lin Padgham, David C. Parkes, Jörg P. Müller, and Simon Parsons, editors, 7th International Joint Conference on Autonomous Agents and Multiagent Systems (AAMAS 2008), Estoril, Portugal, May 12-16, 2008, Volume 2, pages 1015-1022. IFAAMAS, 2008.

14. Dov Gabbay, John Horty, Xavier Parent, Ron van der Meyden, and Leendert van der Torre, editors. Handbook of Deontic Logic and Normative Systems. College Publications, London, 2014.

15. Davide Grossi, Luca Tummolini, and Paolo Turrini. Norms in game theory. In Handbook of Agreement Technologies. 2012.

16. Paul Harrenstein. Logic in conflict. PhD thesis, Utrecht University, 2004.

17. Paul Harrenstein, Wiebe van der Hoek, John-Jules Meyer, and Cees Witteveen. Boolean games. In Proceedings of the 8th Conference on Theoretical Aspects of Rationality and Knowledge, TARK '01, pages 287-298, San Francisco, CA, USA, 2001. Morgan Kaufmann Publishers Inc.

18. Andreas Herzig, Emiliano Lorini, Frédéric Moisan, and Nicolas Troquard. A dynamic logic of normative systems. In Toby Walsh, editor, IJCAI 2011, Proceedings of the 22nd International Joint Conference on Artificial Intelligence, Barcelona, Catalonia, Spain, July 16-22, 2011, pages 228-233. IJCAI/AAAI, 2011.

19. Leonid Hurwicz. Institutions as families of game forms. Japanese Economic Review, 47(2):113-132, 1996.

20. Leonid Hurwicz. But who will guard the guardians? American Economic Review, 98(3):577-585, 2008.

21. David Makinson and Leendert van der Torre. Input-output logics. Journal of Philosophical Logic, 29:383-408, 2000. 
22. David Makinson and Leendert van der Torre. Permission from an input/output perspective. Journal of Philosophical Logic, 32:391-416, 2003.

23. Marios Mavronicolas, Burkhard Monien, and Klaus W. Wagner. Weighted boolean formula games. In Xiaotie Deng and Fan Chung Graham, editors, Internet and Network Economics, Third International Workshop, WINE 200\%, San Diego, CA, USA, December 12-14, 2007, Proceedings, volume 4858 of Lecture Notes in Computer Science, pages 469-481. Springer, 2007.

24. Martin Osborne and Ariel Rubinstein. A Course in Game Theory. The MIT Press, Cambridge, Mass., 1994.

25. Xavier Parent. Moral particularism in the light of deontic logic. Artif. Intell. Law, 19(2-3):75-98, 2011.

26. Luigi Sauro and Serena Villata. Dependency in cooperative boolean games. J. Log. Comput., 23(2):425-444, 2013.

27. Yoav Shoham and Kevin Leyton-Brown. Multiagent Systems - Algorithmic, GameTheoretic, and Logical Foundations. Cambridge University Press, 2009.

28. Xin Sun. How to build input/output logic. In Nils Bulling, Leendert W. N. van der Torre, Serena Villata, Wojtek Jamroga, and Wamberto Weber Vasconcelos, editors, Computational Logic in Multi-Agent Systems - 15th International Workshop, CLIMA XV, Prague, Czech Republic, August 18-19, 2014. Proceedings, volume 8624 of Lecture Notes in Computer Science, pages 123-137. Springer, 2014.

29. Edna Ulmann-Margalit. The Emergence of Norms. Clarendon Press,, Oxford, 1977.

30. Georg von Wright. Deontic logic. Mind, 60:1-15, 1952.

31. Gerhard Weiss, editor. Multiagent systems. MIT press, 2 edition, 2013.

32. Michael Wooldridge. An Introduction to MultiAgent Systems (2. ed.). Wiley, 2009. 